\title{
Comparison of lipid accumulation product and body mass index as indicators of diabetes diagnosis among 215,651 Chinese adults
}

\author{
Tian Tian $^{1}$, Pei Hualian ${ }^{1}$, Chen Zhen ${ }^{1}$, Gulisiya Hailili ${ }^{1}$, Wang Shuxia ${ }^{2}$, Sun Yong ${ }^{2}$, Yao Hua ${ }^{\text {Corresp., }}{ }^{2}$, Dai Jianghong \\ Corresp. 1 \\ ${ }^{1}$ School of Public Health, Xinjiang Medical University, Urumqi, China \\ 2 School of Health Management, Xinjiang Medical University, Urumqi, China \\ Corresponding Authors: Yao Hua, Dai Jianghong \\ Email address: yaohua01@sina.com, epidjh@163.com
}

Purpose. We aimed to assess if lipid accumulation product (LAP) could outperform body mass index (BMI) as a marker for diabetes diagnosis. Methods. We analyzed the results of a national physical examination project in Urumqi, China. This project was conducted in 442 community clinics in Urumqi from October 2016 to February 2017. Results. LAP was highly correlated with diabetes. The subjects with higher amounts of LAP had a higher risk of diabetes, and the prevalence of diabetes in the fourth quartile of LAP was dramatically higher than in the first quartile (5.72\% vs. $21.76 \%)$. The adjusted odds ratios (AOR) associated with diabetes in the fourth quartile of LAP was significantly higher than the AOR associated with diabetes in the first quartile, and when $\mathrm{BMI} \geq 28 \mathrm{~kg} / \mathrm{m}^{2}$ was compared with $\mathrm{BMI}<28 \mathrm{~kg} / \mathrm{m}^{2}[3.24(3.11,3.37)$ vs. 1.65(1.60,1.70)]. The LAP's area under the curve (AUC) was significantly higher than the BMI's AUC when based on diabetes (0.655 vs. 0.604$)$. In the normal BMI group, $34 \%$ of participants had a LAP value higher than the cut-off point found during ROC analysis. In this subgroup, we observed a significantly higher prevalence of diabetes that was similar to that of the subgroup with a $\mathrm{BMI} \geq 28 \mathrm{~kg} / \mathrm{m}^{2}$, and both of their LAP values were higher than the cut-off point. Conclusion. When use as a tool for diabetes diagnosis, LAP performed better than BMI, implying that LAP could be a preferable anthropometry assessment. 


\section{Comparison of lipid accumulation product and body}

2 mass index as indicators of diabetes diagnosis among

3 215,651 Chinese adults

4 Tian Tian ${ }^{1}$, Pei Hualian ${ }^{1}$, Chen Zhen ${ }^{1}$, Gulisiya Hailili' ${ }^{1}$, Wang Shuxia ${ }^{2}$, Sun Yong ${ }^{2}$, Yao

5 Hua $^{2^{\star}}$, Dai Jianghong ${ }^{1^{*}}$

6 'School of Public Health, Xinjiang Medical University, Urumqi, Xinjiang, China

7 2School of Health Management, Xinjiang Medical University, Urumqi, Xinjiang, China

8 Corresponding Author:

9 Yao Hua ${ }^{2}$

10 Xinjiang, China

11 E-mail address: yaohua01@sina.com

12 Dai Jiang Hong ${ }^{1}$

13 Xinjiang, China

14 E-mail address: epidjh@163.com

\section{Abstract}

Purpose. We aimed to assess if lipid accumulation product (LAP) could outperform body

17 mass index (BMI) as a marker for diabetes diagnosis.

18 Methods. We analyzed the results of a national physical examination project in Urumqi,

19 China. This project was conducted in 442 community clinics in Urumqi from October 202016 to February 2017.

21 Results. LAP was highly correlated with diabetes. The subjects with higher amounts of 22 LAP had a higher risk of diabetes, and the prevalence of diabetes in the fourth quartile 23 of LAP was dramatically higher than in the first quartile $(5.72 \%$ vs. $21.76 \%)$. The 24 adjusted odds ratios (AOR) associated with diabetes in the fourth quartile of LAP was 25 significantly higher than the AOR associated with diabetes in the first quartile, and when 
$26 \mathrm{BMI} \geq 28 \mathrm{~kg} / \mathrm{m}^{2}$ was compared with $\mathrm{BMl}<28 \mathrm{~kg} / \mathrm{m}^{2}[3.24(3.11,3.37)$ vs. $1.65(1.60,1.70)]$.

27 The LAP's area under the curve (AUC) was significantly higher than the BMl's AUC

28 when based on diabetes ( 0.655 vs. 0.604$)$. In the normal BMI group, $34 \%$ of participants

29 had a LAP value higher than the cut-off point found during ROC analysis. In this

30 subgroup, we observed a significantly higher prevalence of diabetes that was similar to

31 that of the subgroup with a BMl $\geq 28 \mathrm{~kg} / \mathrm{m}^{2}$, and both of their LAP values were higher

32 than the cut-off point.

33 Conclusion. When use as a tool for diabetes diagnosis, LAP performed better than BMI,

34 implying that LAP could be a preferable anthropometry assessment.

\section{Introduction}

36 Over the past years, the prevalence of type 2 diabetes has increased considerably worldwide, with more notable increases in developing countries [1]. China, the largest middle-income country, has the largest diabetes epidemic in the world, with a prevalence of $10.9 \%$ in 2013 [2]. Diabetes is linked with a number of vascular and nonvascular complications. [3].

41 The pathogen of diabetes is not well defined, but inducing factors include genetics, inflammation, aging, lifestyle, and obesity [4]. The risk of diabetes in adults increases with increasing adiposity, and decreases with fat loss. Adiposity leads to insulin resistance, is connected to molecules that make individuals susceptible to inflammation and metabolic complications [5], and is believed to be a promoter of type 2 diabetes mellitus. There are a number of techniques designed to estimate obesity, such as underwater weighing, dual-energy X-ray absorption (DXA), computed tomography scans and magnetic resonance imaging. These measurements have their own advantages -- while some are most precise for measuring body fat, others can evaluate body fat distribution [6-9]. However, these technologically complicated approaches are too expensive and time-consuming to be applied in large-scale investigations. 
52 In the past few years, anthropometric indicator measurements such as body mass index

53 (BMI) have been recognized as cost-effective ways to evaluate obesity. BMI typically represents overall obesity, while other measurements are applied to indicate central obesity. However, BMI is now being cautioned as an inadequate indicator of individual obesity, and an increasing number of studies have suggested that central adiposity may be a better indicator of diabetes mellitus (Browning et al. 2010; Lee et al. 2008; Park et al. 2009; Taylor et al. 2010; Tseng et al. 2010). For central adiposity, waist circumference (WC) has been considered as a lucid estimation. Motamed found a notable association between triglyceride (TG) level and risk of metabolic syndrome(Motamed et al. 2016). Furthermore, there is proof that increased WC is associated with the aggregation of TG levels. A number of studies have shown that contemporaneous quantification of TG levels and WC could indicate a symptom of lipid overaccumulation related to the risk of metabolic syndrome. In 2005, Henry S Kahn described a simple index, counting as the compound of WC and fasting plasma TG levels, in order to estimate excessive lipid accumulation in adults. He utilized the lipid overaccumulation concept, and tested the assumption that the mentioned index is connected with various cardiovascular risk factors more effectively than BMI(Kahn 2005).

An increasing number of studies view LAP as an effective marker for diabetes(Bozorgmanesh et al. 2010), metabolic syndrome(Motamed et al. 2016; Taverna et al. 2011), and insulin resistance(Xia et al. 2012) among the overall population. These findings inspired us to test the relationship between LAP and diabetes in a large-scale population. Our major point in this research was to evaluate whether LAP is better than BMI as an indicator of diabetes among the overall population, and to find out its optimal cutoff point for diabetes diagnosis. 
78

79

80

81

82

83

84

85

86

87

88

89

90

91

92

93

94

95

96

97

98

100

101

102

\section{Population}

Beginning in September 2016, Xinjiang, the largest autonomous region in China, launched a comprehensive physical examination project within the region. All residents in Xinjiang can participate in this free annual physical examination, with the cost covered by the local government. The residents were recruited by government announcement. Urumqi, the capital of Xinjiang, is the center of Silk Road economic zone, and understanding the health status of the citizens of Urumqi is one of the most important purposes of the national physical examination project. From October 2016 to February 2017, 303,620 subjects in Urumqi participated in this physical examination project. According to the sixth census and the projection of the annual report of the Public Security Bureau, there were 3.12 million permanent residents in Urumqi in 2016. The Medical Ethics Committee of the Fourth Affiliated Hospital of Xinjiang Medical University approved the study protocol (2018XE0108). Informed written consent was obtained from all subjects.

2 The present research examined data from Urumqi. Participants over 18 years qualified

93 for the current analysis. Participants who had incomplete information on variables (waist 4 circumference, fasting plasma triglyceride, height, weight) used to calculate LAP and

BMI were excluded. The final sample of this analysis consisted of 215,651 participants.

\section{Data collection and laboratory tests}

Four hundred and forty-two community clinics in Urumqi were involved in this national physical examination project. All community clinic staff involved in this project were trained on the protocol established by the National Health Commission of Xinjiang. The training included questionnaires, physical measurements, laboratory tests, and data entry. Participants were interviewed in the community health center closest to their house, and the interview location for each participant was recorded. Trained 
103 interviewers use pre-tested questionnaires to gather information. The collected

104 information included demographic data and behavior risk factors.

105 Weight was valued using digital scales with participants wearing as few clothes as

106 possible without shoes, and the weight reading was accurate to $100 \mathrm{~g}$. Height was

107 valued by a tape meter in the standing position, without shoes and with the shoulders

108 aligned normally. When measuring the waist circumference, an unstretched tape meter

109 was kept at the umbilical level and recorded to the nearest $0.1 \mathrm{~cm}$, making sure that

110 there was no pressure on the body surface. All the participants wore as few clothes as

111 possible.

112 After the subjects were fasted for at least 8 hours, the investigators collected $5 \mathrm{~mL}$ of

113 blood samples from the subjects and placed blood samples in vacutainer tubes of EDTA

114 (ethylenediaminetetraacetic acid) for determination of glucose and other indices. Serum

115 concentrations of TG, fasting glucose, serum total cholesterol and high-density

116 lipoprotein (HDL) were tested through a biochemical analyzer (Dimension AR/AVL

117 Clinical Chemistry System, Newark, NJ, USA) in the Laboratory of the community clinic

118 where the participant had been interviewed.

\section{Definition of variables and outcomes}

$120 \mathrm{BMI}\left(\mathrm{kg} / \mathrm{m}^{2}\right)$ is equal to body weight $(\mathrm{kg})$ divided by the square of the height $(\mathrm{m})$.

121 According to the Chinese definitions, participants whose $B M l \geq 28 \mathrm{~kg} / \mathrm{m}^{2}$ were defined as

122 obese(Zhou 2002). For men, LAP = [WC $(\mathrm{cm})-65] \times[\mathrm{TG}(\mathrm{mmol} / \mathrm{L})]$; for women, LAP =

$123[\mathrm{WC}(\mathrm{cm})-58] \times[\mathrm{TG}(\mathrm{mmol} / \mathrm{L})]$. A diabetes diagnosis was defined as having a history of

124 diabetes mellitus, if they were using antidiabetic agents currently, or a FPG level $\geq 7.0$

$125 \mathrm{mmol} / \mathrm{l} .(2006)$

126 Statistical analysis 
127 The categorical variables of participants' demographic characteristics were presented in

128 numbers and percentage. Continuous variables were displayed in mean \pm standard

129 deviation (SD). For categorical variables, we used Pearson's $X^{2}$ test to compare basic

130 characteristics of different diabetes statuses (with/without), and for continuous variables,

131 we used Student's t-tests. In order to get a profound understanding of the connection

132 between LAP levels and the epidemic of diabetes, we split the study participants into

133 four groups according to LAP quartiles. We used Pearson's $\mathrm{x} 2$ test to compare the

134 discrepancy of diabetes prevalence in different groups. To calculate the odds ratios

135 (ORs) with 95\% confidence intervals (Cls) and adjusted odds ratios (AORs) with 95\%

136 confidence intervals (Cls), we used logistic regression, and then the ORs and AORs

137 were used to estimate the risk of diabetes in every LAP quartile, setting the lowest

138 quartile of LAP as reference. Age, gender, education, smoking and alcohol consumption

139 were all entered into the multivariate logistic model to calculate the AOR. We calculated

140 the area under the curve (AUC) using receiver operating characteristics (ROC), and the

141 AUC was used to estimate the intensity of the relationship between subdivided LAP

142 level and diabetes. At the highest Youden Index [(specificity + sensibility) - 1], the best

143 cut-off point was determined, and the discrimination among different AUCs was tested

144 by a nonparametric method. We used R 3.4 .1 for all the analyses and $\mathrm{P}$ values of $<0.05$

145 (two-tailed) were considered as statistically significant.

\section{Results}

\section{Characteristics of the study subjects}

148 During the study, 215,651 Chinese subjects were included. The characteristics of all 149 participants, with and without diabetes, are outlined in Table 1. The mean age of all

150 subjects was 50.02 years old. Of them, $55.86 \%$ were female, $34.71 \%$ had received a

151 junior middle school education, $16.72 \%$ were current smokers, and $16.09 \%$ were

152 current drinkers. Among the 215,651 subjects, 27,917 had diabetes, and the prevalence 
153 of diabetes was $12.95 \%$. Patients with diabetes were older and had higher weights and

154 TG levels than those subjects without diabetes (Table 1).

155 Association between lipid accumulation product and diabetes

156 To more efficiently uncover the relationship between LAP and the prevalence of

157 diabetes, the participants were divided into four subgroups based on LAP level.

158 According to the survey, the prevalence of diabetes was greater in the higher LAP

159 quartile groups. We also divided the subjects according to their BMI, and the prevalence

160 of diabetes was expressively higher in the subjects who had higher BMI (Table 2).

161 The non-adjusted and adjusted analysis association among quartiles of LAP/BMI are

162 shown in Table 3. In the multiple logistic regression model, confounding factor including

163 age, education, smoking and alcohol consumption were entered into the model to calculate

164 the AOR, and we did not eliminate any variables. Compared to the first quartile of LAP,

165 the COR associated with diabetes of the second [OR $(95 \% \mathrm{Cl}): 1.28(1.22,1.34)]$, third

166 [OR $(95 \% \mathrm{Cl}): 1.86(1.78,1.95)]$ and fourth quartile [OR $(95 \% \mathrm{Cl}): 4.67(4.49,4.86)]$ of

167 LAP were significant; the COR associated with diabetes for a BMI $\geq 28 \mathrm{~kg} / \mathrm{m}^{2}$ [OR $(95 \%$

$168 \mathrm{Cl}): 1.65(1.63,1.74)]$ was significant when compared with a $\mathrm{BMl}<28 \mathrm{~kg} / \mathrm{m}^{2}$. Compared

169 with the first quartile, the risks associated with diabetes for the third and last quartiles of

170 LAP were both still significant after adjusted age, gender, education, smoking status

171 and alcohol consumption. The AORs $(95 \% \mathrm{Cl})$ were $0.97(0.92,1.02), 1.28(1.23,1.34)$

172 and 3.24(3.11,3.37) for the second, third and last quartiles of LAP, respectively.

173 Meanwhile, the AOR associated with diabetes for a BMI $\geq 28 \mathrm{~kg} / \mathrm{m}^{2}$ [OR $(95 \% \mathrm{Cl})$ :

$1741.65(1.60,1.70)]$ was also still significant when compared with a BMl<28 kg/m² (Table $1753)$.

176 Evaluation of the predictive accuracy of LAP and BMI

177 LAP exhibited higher diagnostic accuracy for diabetes compared to BMI $(p<0.0001)$. 
178 For LAP, the optimum cutoff points were 38.045 (sensitivity $=60.6 \%$, specificity $=62.0$

$179 \%$ ), while for BMI, the optimum cutoff points were 25.065 (sensitivity $=55.2 \%$, specificity $180=60.2 \%)($ Table 4$)$.

\section{Distribution of LAP according to BMI}

182 After divided according to BMI ( $<28 \mathrm{~kg} / \mathrm{m}^{2}$ or $\left.\geq 28 \mathrm{~kg} / \mathrm{m}^{2}\right)$, the participants were

183 subdivided into two groups according to the cut-off point of LAP obtained in this study,

184 and the distribution of LAP values in subjects with and without diabetes were compared.

185 In the normal BMI group, $34 \%$ of subjects had a LAP higher than the cut-off point, and

186 their prevalence of diabetes was significantly higher than the group whose LAP was

187 below the cut-off value. We also compared the distribution of lap among males and

188 females, the results indicated that there was significantly different $(P<0.001)$ (Table 5).

\section{Discussion}

190 Obesity has received extensive attention as a risk factor for diabetes in recent years,

191 but there is still controversy about which obesity index performs better in a population

192 study. LAP is uncomplicated, cheap, and could be useful when height and/or weight are

193 difficult to assess (such as, when a person has lost a limb). In our study, we analyzed

194 data from a national physical examination project, tried to determine the relationship

195 between LAP and diabetes, and compared LAP with BMI for diabetes diagnostic

196 accuracy. The results indicated that LAP strongly correlated with diabetes. The subjects

197 with higher LAP had a higher risk of diabetes, the prevalence of diabetes in the fourth

198 quartile of LAP was dramatically higher than in the first quartile (5.72\% vs. $21.76 \%)$, and

199 the conclusion was consistent with other studies. For example, Shen YY conducted a

200 cross-sectional study in Beijing, the capital of China, and concluded that an elevated

201 level of LAP was linked to an increased risk of diabetes in adults, and that LAP could be

202 an key predictor for diabetes (Shen et al. 2017). Wakabayashi I and his team carried out 
203 a number of studies to confirm the relationship between LAP and metabolic diseases

204 such as diabetes and atherosclerosis. All his surveys showed a positive correlation, 205 while some of his studies had taken the age and gender of subjects into consideration.

206 (Wakabayashi 2014; Wakabayashi \& Daimon 2012; Wakabayashi \& Daimon 2014)

207 The AOR associated with diabetes in the fourth quartile of LAP when compared with the 208 first quartile was significantly higher than the AOR of $B M I \geq 28 \mathrm{~kg} / \mathrm{m}^{2}$ when compared 209 with a BMl<28 kg/m²[3.24(3.11,3.37) vs. 1.65(1.60,1.70)], and LAP's AUC based on

210 diabetes was significantly higher than BMl's AUC based on diabetes (0.655 vs. 0.604).

211 This shows that LAP has a stronger association with diabetes than BMI does. A number

212 of studies have found similar results. An investigation conducted among Mongolians in

213 China compared the relationship of LAP and BMI with the risk of hypertension, and they

214 found LAP was better than BMI. (Gao et al. 2013)Xia C implied that LAP was a more

215 effective indicator than WC and BMI when identifying insulin resistance in non-diabetic

216 individuals. (Gao et al. 2013) Assessment of the predictive accuracy of LAP and BMI

217 also confirmed that LAP is a better obesity index than BMI for the diagnosis of diabetes.

218 This is not only supported by the data in our survey, but also by other research that has

219 evaluated LAP and BMl's diagnostic sensitivity and specificity for metabolic disease

220 (Motamed et al. 2016; Taverna et al. 2011).

221 BMI is treated as a scale of overweight and obesity, but should be used conservatively

222 in epidemiological studies to estimate the health risk caused by the overaccumulation of

223 body fat (Kishida et al. 2012). Subjects of different ages, sex and ethnicities can show

224 high variability or may have the same BMI when we use BMI to interpret overweight and

225 obese body composition. Overaccumulation of body fat in the viscera presents an

226 additional health risk, but BMI does not imply any thorough understanding of partial

227 body fat distribution and accumulation. In our study, after finding the optimal cutoff

228 points, we found that in the normal BMI group, $34 \%$ of participants still had a LAP value 
229 higher than the cut-off point, and the prevalence of diabetes was significantly higher

230 than in the other subgroups It was even close to the subgroup whose $B M I \geq 28 \mathrm{~kg} / \mathrm{m}^{2}$

231 and whose LAP value was higher than the cut-off point. Thus, BMI is not an accurate

232 scale of body composition and distribution in individuals. (Prentice \& Jebb 2001) WC is

233 an important additional piece of information for assessing abdominal obesity that may

234 better explain obesity-related health risk (Janssen et al. 2004). It is easy to measure

235 and shows high stability between different surveys (Wang et al. 2003). Although it is

236 better to use WC to evaluate total abdominal fat, WC cannot make a distinction between

237 visceral adiposity and subcutaneous abdominal fat, while fat accumulation in the viscera

238 is highly correlated with metabolic abnormalities. (Despres 2006; Kishida et al. 2012)

239 Serum triglyceride level, even within the accepted normal range, is an independent risk

240 factor for impaired fasting glucose and diabetes mellitus. LAP is an obesity index

241 combining WC and TG together, so it is quite reasonable that it outperforms $\mathrm{BMI}$ in

242 diagnosing diabetes and other metalogic diseases.

243 This large-scale project also allowed us to understand the overall prevalence and

244 distribution of diabetes and other chronic diseases in Urumqi. The latest countrywide

245 typical cross-sectional study in mainland China was conducted in 2013, which included

246170,287 subjects, and concluded that the prevalence of diabetes was $10.9 \%$. They

247 estimated that the prevalence of diabetes in underdeveloped areas was $9.6 \%$, although

248 Urumqi, where our study conducted, is a typical underdeveloped city, and our result was

249 significantly higher at $12.95 \%$. We assumed that this discrepancy in diabetes

250 prevalence was due to the participant criteria, as the previous study designed a

251 multistage sampling method(Wang et al. 2017; Xu et al. 2013; Zhou et al. 2015), while

252 we recruited volunteer subjects to join this project, implying that the participants in our

253 study were more likely to be in poor physical conditions. This also implies that our

254 research may overestimate the prevalence of diabetes in Urumqi, but the focus of our 
255 research is in the comparison of the diagnostic value of LAP and BMI of diabetes. This

256 overestimation does not seem to have a substantive impact on our conclusion.

257 We should mention our study has several limitations. First of all, we did not have data to 258 compare the participants to non-participants, but during the project, we found that most

259 of the participants were not covered by other physical examination project, which means

260 them were poorer than the non-participants. Second, as a cross-sectional study, and

261 thus a causal association between LAP and diabetes cannot be determined. We

262 encourage additional longitudinal studies to confirm our conclusions.

\section{Conclusions}

264 In summary, this study indicated that LAP could be a better anthropometric

265 measurement to diagnosis diabetes. In the future, prospective cohort studies should be

266 conducted to test the causal association between LAP and diabetes.

\section{Reference}

268 2006. Global Guideline for Type 2 Diabetes: recommendations for standard, comprehensive, and

269 minimal care. Diabet Med 23:579-593. 10.1111/j.1464-5491.2006.01918.x

270 Bozorgmanesh M, Hadaegh F, and Azizi F. 2010. Diabetes prediction, lipid accumulation product, 271 and adiposity measures; 6-year follow-up: Tehran lipid and glucose study. Lipids Health Dis 9:45.

272 10.1186/1476-511x-9-45

273 Browning LM, Hsieh SD, and Ashwell M. 2010. A systematic review of waist-to-height ratio as a

274 screening tool for the prediction of cardiovascular disease and diabetes: 0.5 could be a suitable

275 global boundary value. Nutr Res Rev 23:247-269. 10.1017/s0954422410000144

276 Despres JP. 2006. Is visceral obesity the cause of the metabolic syndrome? Ann Med 38:52-63.

277 10.1080/07853890500383895

278 Gao X, Wang G, Wang A, Xu T, Tong W, and Zhang Y. 2013. Comparison of lipid accumulation

279 product with body mass index as an indicator of hypertension risk among Mongolians in China.

280 Obes Res Clin Pract 7:e308-314. 10.1016/j.orcp.2012.02.002

281 Janssen I, Katzmarzyk PT, and Ross R. 2004. Waist circumference and not body mass index

282 explains obesity-related health risk. Am J Clin Nutr 79:379-384. 10.1093/ajen/79.3.379

283 Kahn HS. 2005. The "lipid accumulation product" performs better than the body mass index for 284 recognizing cardiovascular risk: a population-based comparison. BMC Cardiovasc Disord 5:26.

$285 \quad 10.1186 / 1471-2261-5-26$ 
286 Kishida K, Funahashi T, Matsuzawa Y, and Shimomura I. 2012. Visceral adiposity as a target for

287 the management of the metabolic syndrome. Ann Med 44:233-241.

$288 \quad 10.3109 / 07853890.2011 .564202$

289 Lee CM, Huxley RR, Wildman RP, and Woodward M. 2008. Indices of abdominal obesity are

290 better discriminators of cardiovascular risk factors than BMI: a meta-analysis. J Clin Epidemiol

291 61:646-653. 10.1016/j.jclinepi.2007.08.012

292 Motamed N, Razmjou S, Hemmasi G, Maadi M, and Zamani F. 2016. Lipid accumulation product

293 and metabolic syndrome: a population-based study in northern Iran, Amol. J Endocrinol Invest

294 39:375-382. 10.1007/s40618-015-0369-5

295 Park SH, Choi SJ, Lee KS, and Park HY. 2009. Waist circumference and waist-to-height ratio as

296 predictors of cardiovascular disease risk in Korean adults. Circ J 73:1643-1650.

297 Prentice AM, and Jebb SA. 2001. Beyond body mass index. Obes Rev 2:141-147.

298 Shen YY, Chen JC, Li G, Cao J, Li JX, Huang JF, and Gu DF. 2017. [Relationship of lipid

299 accumulation product with hypertension and diabetes among Beijing residents study]. Zhonghua

300 Yu Fang Yi Xue Za Zhi 51:415-420. 10.3760/cma.j.issn.0253-9624.2017.05.009

301 Taverna MJ, Martinez-Larrad MT, Frechtel GD, and Serrano-Rios M. 2011. Lipid accumulation

302 product: a powerful marker of metabolic syndrome in healthy population. Eur J Endocrinol

303 164:559-567. 10.1530/eje-10-1039

304 Taylor AE, Ebrahim S, Ben-Shlomo Y, Martin RM, Whincup PH, Yarnell JW, Wannamethee SG, 305 and Lawlor DA. 2010. Comparison of the associations of body mass index and measures of central 306 adiposity and fat mass with coronary heart disease, diabetes, and all-cause mortality: a study using 307 data from 4 UK cohorts. Am J Clin Nutr 91:547-556. 10.3945/ajcn.2009.28757

308 Tseng CH, Chong CK, Chan TT, Bai CH, You SL, Chiou HY, Su TC, and Chen CJ. 2010. Optimal 309 anthropometric factor cutoffs for hyperglycemia, hypertension and dyslipidemia for the Taiwanese 310 population. Atherosclerosis 210:585-589. 10.1016/j.atherosclerosis.2009.12.015

311 Wakabayashi I. 2014. Influence of age and gender on lipid accumulation product and its relation

312 to diabetes mellitus in Japanese. Clin Chim Acta 431:221-226. 10.1016/j.cca.2014.02.002

313 Wakabayashi I, and Daimon T. 2012. Receiver-operated characteristics (ROCs) of the 314 relationships between obesity indices and multiple risk factors (MRFs) for atherosclerosis at 315 different ages in men and women. Arch Gerontol Geriatr 55:96-100.

316 10.1016/j.archger.2011.06.028

317 Wakabayashi I, and Daimon T. 2014. A strong association between lipid accumulation product 318 and diabetes mellitus in japanese women and men. J Atheroscler Thromb 21:282-288.

319 Wang J, Thornton JC, Bari S, Williamson B, Gallagher D, Heymsfield SB, Horlick M, Kotler D,

320 Laferrere B, Mayer L, Pi-Sunyer FX, and Pierson RN, Jr. 2003. Comparisons of waist 321 circumferences measured at 4 sites. Am J Clin Nutr 77:379-384. 10.1093/ajcn/77.2.379

322 Wang L, Gao P, Zhang M, Huang Z, Zhang D, Deng Q, Li Y, Zhao Z, Qin X, Jin D, Zhou M, 323 Tang X, Hu Y, and Wang L. 2017. Prevalence and Ethnic Pattern of Diabetes and Prediabetes in 324 China in 2013. Jama 317:2515-2523. 10.1001/jama.2017.7596 
325 Xia C, Li R, Zhang S, Gong L, Ren W, Wang Z, and Li Q. 2012. Lipid accumulation product is a 326 powerful index for recognizing insulin resistance in non-diabetic individuals. Eur J Clin Nutr 327 66:1035-1038. 10.1038/ejcn.2012.83

328 Xu Y, Wang L, He J, Bi Y, Li M, Wang T, Wang L, Jiang Y, Dai M, Lu J, Xu M, Li Y, Hu N, Li 329 J, Mi S, Chen CS, Li G, Mu Y, Zhao J, Kong L, Chen J, Lai S, Wang W, Zhao W, and Ning G. 330 2013. Prevalence and control of diabetes in Chinese adults. Jama 310:948-959. 331 10.1001/jama.2013.168118

332 Zhou BF. 2002. Predictive values of body mass index and waist circumference for risk factors of 333 certain related diseases in Chinese adults--study on optimal cut-off points of body mass index and 334 waist circumference in Chinese adults. Biomed Environ Sci 15:83-96.

335 Zhou M, Astell-Burt T, Bi Y, Feng X, Jiang Y, Li Y, Page A, Wang L, Xu Y, Wang L, Zhao W, 336 and Ning G. 2015. Geographical variation in diabetes prevalence and detection in china: multilevel 337 spatial analysis of 98,058 adults. Diabetes Care 38:72-81. 10.2337/dc14-1100 


\section{Table 1 (on next page)}

Table 1 Characteristics of the study subjects with and without diabetes 
Table 1 Characteristics of the study subjects with and without diabetes

\begin{tabular}{|c|c|c|c|}
\hline Variables & $\begin{array}{c}\text { With diabetes } \\
\text { N (\%) }\end{array}$ & $\begin{array}{c}\text { Without diabetes } \\
\qquad \mathrm{N}(\%)\end{array}$ & $P$ value \\
\hline $\operatorname{Age}^{*}($ year, $\bar{x} \pm s)$ & $58.14 \pm 12.93$ & $48.81 \pm 14.83$ & $<0.001$ \\
\hline Gender & & & $<0.001$ \\
\hline Male & $13472(14.1)$ & $81722(85.9)$ & \\
\hline Female & $14445(12.0)$ & $106012(88.0)$ & \\
\hline Education ${ }^{\#}$ & & & $<0.001$ \\
\hline Illiteracy & 1902(19.3) & 7967(80.7) & \\
\hline Primary school & $7064(15.3)$ & $39225(84.7)$ & \\
\hline Junior high school & $9746(13.0)$ & $65122(87.0)$ & \\
\hline Senior high school & $5519(12.2)$ & $39784(87.8)$ & \\
\hline University or higher & $2922(8.7)$ & $27580(91.3)$ & \\
\hline Unspecified & $764(8.5)$ & $8045(91.5)$ & \\
\hline Height $(\mathrm{cm}, \bar{x} \pm s)$ & $163.3 \pm 8.6$ & $164.0 \pm 8.2$ & $<0.001$ \\
\hline Weight $(\mathrm{kg}, \bar{x} \pm s)$ & $69.9 \pm 11.8$ & $67.0 \pm 11.9$ & $<0.001$ \\
\hline $\mathrm{WC}(\mathrm{cm}, \bar{x} \pm s)$ & $90.6 \pm 10.5$ & $85.8 \pm 10.8$ & $<0.001$ \\
\hline $\mathrm{FPG}(\mathrm{mmol} / \mathrm{L}, \bar{x} \pm s)$ & $7.74 \pm 2.80$ & $5.10 \pm 0.90$ & $<0.001$ \\
\hline $\mathrm{TG}(\mathrm{mmol} / \mathrm{L}, \bar{x} \pm s)$ & $2.07 \pm 1.66$ & $1.57 \pm 1.15$ & $<0.001$ \\
\hline $\mathrm{HDL}-\mathrm{C}(\mathrm{mmol} / \mathrm{L}, \bar{x} \pm s)$ & $1.50 \pm 0.62$ & $1.49 \pm 0.56$ & $<0.001$ \\
\hline Smoking status \& & & & $<0.001$ \\
\hline Non-smokers & $21254(12.3)$ & $151163(87.7)$ & \\
\hline Previous smokers & $1483(21.4)$ & $5438(78.6)$ & \\
\hline Current smokers & $5128(14.2)$ & $30919(85.8)$ & \\
\hline Alcohol consumption $^{\wedge}$ & & & $<0.001$ \\
\hline No & $22564(12.5)$ & $158173(87.5)$ & \\
\hline Yes & $5305(15.3)$ & 29401(84.7) & \\
\hline
\end{tabular}

There were missing values on participants' age, education, smoking status, alcohol consumption, and the number of missing were 353, 11, 266, 208 respectively.

Abbreviations: WC waist circumference, FBG fasting blood glucose, TG triglyceride, HDL-C high-density lipoprotein cholesterol. 
Table 2 (on next page)

Table 2 Distribution of LAP and BMI among subjects with and without diabetes 
Table 2 Distribution of LAP and BMI among subjects with and without diabetes

\begin{tabular}{lrrr}
\hline \multicolumn{1}{c}{ Variables } & $\begin{array}{c}\text { With diabetes } \\
\mathrm{N}(\%)\end{array}$ & $\begin{array}{c}\text { Without diabetes } \\
\mathrm{N}(\%)\end{array}$ & $P$ value \\
\hline Quartiles of LAP & & & $<0.001$ \\
1st $(\leqslant 18.64)$ & $3127(5.7)$ & $51509(94.3)$ & \\
2nd $(18.65 \sim 32.95)$ & $5291(10.0)$ & $47869(90.0)$ & \\
3rd $(32.96 \sim 55.67)$ & $7928(14.7)$ & $46007(85.3)$ & \\
4th $(\geqslant 55.68)$ & $11571(21.5)$ & $42349(78.5)$ & \\
BMI $\left(\mathrm{kg} / \mathrm{m}^{2}\right)$ & & & \\
$<28$ & $20229(11.7)$ & $152470(88.3)$ & $<0.001$ \\
$\geqslant 28$ & $7688(17.9)$ & $35264(82.1)$ & \\
\hline
\end{tabular}

1 


\section{Table 3(on next page)}

Table 3 Odds ratios and 95\% confidence intervals for diabetes according to quartiles of LAP and BMI 
Table 3 Odds ratios and 95\% confidence intervals for diabetes according to quartiles of LAP and BMI

\begin{tabular}{llllr}
\hline Variables & $C O R(95 \% C I)$ & $P$ value & $A O R(95 \% C I)$ & $P$ value \\
\hline Quartiles of LAP & & & & \\
1st $(\leqslant 18.64)$ & & & & \\
2nd $(18.65 \sim 32.95)$ & $1.28(1.22 \sim 1.34)$ & $<0.001$ & $0.97(0.92 \sim 1.02)$ & 0.24 \\
3rd $(32.96 \sim 55.67)$ & $1.86(1.78 \sim 1.95)$ & $<0.001$ & $1.28(1.23 \sim 1.34)$ & $<0.001$ \\
4th $(\geqslant 55.68)$ & $4.67(4.49 \sim 4.86)$ & $<0.001$ & $3.24(3.11 \sim 3.37)$ & $<0.001$ \\
BMI $\left(\mathrm{kg} / \mathrm{m}^{2}\right)$ & & & & \\
$\quad<28$ & & & & \\
$\geqslant 28$ & $1.65(1.63 \sim 1.74)$ & $<0.001$ & $1.65(1.60 \sim 1.70)$ & $<0.001$ \\
\hline
\end{tabular}

1 COR: crude odds ratio; $A O R$ : adjusted odds ratio, 95\% CI: 95\% confidence interval. Age, gender, education, 2 smoking and alcohol consumption were adjusted for calculated AOR 


\section{Table 4 (on next page)}

Table 4 Assessment of the predictive accuracy of LAP and BMI by gender 
Table 4 Assessment of the predictive accuracy of LAP and BMI by gender

\begin{tabular}{llllcc}
\hline \multicolumn{2}{l}{ Variables } & AUC $(95 \% \mathrm{CI})$ & Cut-off & Sensitivity (\%) & Specificity (\%) \\
\hline \multirow{2}{*}{ Total } & LAP & $0.655(0.652 \sim 0.658)$ & 38.41 & 60.6 & 62.0 \\
& BMI & $0.604(0.600 \sim 0.607)$ & 25.07 & 60.2 & 55.2 \\
\multirow{4}{*}{ male } & LAP & $0.625(0.621 \sim 0.630)$ & 35.71 & 64.5 & 53.3 \\
& BMI & $0.580(0.576 \sim 0.586)$ & 24.84 & 66.7 & 45.8 \\
\multirow{2}{*}{ female } & LAP & $0.679(0.674 \sim 0.684)$ & 33.05 & 71.1 & 56.1 \\
& BMI & $0.618(0.614 \sim 0.623)$ & 24.23 & 65.8 & 51.9 \\
\hline
\end{tabular}

1 AUC: area under the curve; 
Table 5 (on next page)

Table 5 Distribution of LAP according to gender and BMI 
1

Table 5 Distribution of LAP according to gender and BMI

\begin{tabular}{|c|c|c|c|c|c|}
\hline group & & LAP & $\begin{array}{c}\text { With diabetes } \\
\mathrm{N}(\%)\end{array}$ & $\begin{array}{c}\text { Without diabetes } \\
\mathrm{N}(\%)\end{array}$ & $P$ value \\
\hline \multirow{4}{*}{ Gender* } & \multirow{2}{*}{ male } & $<38.045$ & $5238(10.2)$ & $46140(89.8)$ & $<0.001$ \\
\hline & & $\geqslant 38.045$ & $8234(18.8)$ & $35582(81.2)$ & \\
\hline & \multirow{2}{*}{ female } & $<38.045$ & $5226(7.3)$ & $66626(92.7)$ & $<0.001$ \\
\hline & & $\geqslant 38.045$ & $9219(19.0)$ & $39386(81.0)$ & \\
\hline \multirow{4}{*}{ BMI $\left(\mathrm{kg} / \mathrm{m}^{2}\right)^{\#}$} & \multirow{2}{*}{$<28$} & $<38.045$ & $9409(8.3)$ & $104497(91.7)$ & $<0.001$ \\
\hline & & $\geqslant 38.045$ & $10820(18.4)$ & 47973(81.6) & \\
\hline & \multirow{2}{*}{$\geqslant 28$} & $<38.045$ & $1055(11.4)$ & $8269(88.7)$ & $<0.001$ \\
\hline & & $\geqslant 38.045$ & $6633(19.7)$ & $26995(80.3)$ & \\
\hline
\end{tabular}

2 *The distribution of LAP between males and females was significantly different $(P<0.001)$. The distribution of 3 LAP between BMI $<28 \mathrm{~kg} / \mathrm{m}^{2}$ and BMI $\geqslant 28 \mathrm{~kg} / \mathrm{m}^{2}$ was significantly $\operatorname{different}(P<0.001)$. 